\title{
On Thought Experiments: Is There More to the Argument?
}

\author{
John D. Norton $†$
}

Thought experiments in science are merely picturesque argumentation. I support this view in various ways, including the claim that it follows from the fact that thought experiments can err but can still be used reliably. The view is defended against alternatives proposed by my cosymposiasts.

1. Introduction. A scientist - a Galileo, Newton, Darwin or Einsteinpresents us with some vexing problem. We are perplexed. In a few words of simple prose, the scientist then conjures up an experiment, purely in thought. We follow, replicating its falling bodies or spinning buckets in our minds, and our uncertainty evaporates. We know the resolution and somehow we sense that we knew it all along. That moment of realization is exquisite, and it is difficult to resist the sense that something of profound epistemic moment has just transpired.

My purpose here is to give strength to those who want to resist. My view of thought experiments is quite deflationary. I claim that they are just ordinary argumentation, disguised in some vivid picturesque or narrative form. Therefore they can do nothing more epistemically that can ordinary argumentation. I don't doubt that this picturesque clothing gives them special rhetorical powers, but they are not my concern. More precisely, my concern here is what I label:

The epistemological problem of thought experiments in the sciences. Thought experiments are supposed to give us knowledge of the natural world. From where does this knowledge come?

Since I claim that thought experiments are merely picturesque arguments,

$\dagger$ To contact the author, please write to: Department of History and Philosophy of Science, University of Pittsburgh, Pittsburgh, PA 15260; e-mail: jdnorton@pitt.edu.

$\$$ My thanks to my cosymposiasts, Tamar Gendler, James McAllister, and especially Jim Brown for years of stimulating discussion.

Philosophy of Science, 71 (December 2004) pp. 1139-1151. 0031-8248/2004/7105-0043\$10.00

Copyright 2004 by the Philosophy of Science Association. All rights reserved. 
my solution is that this knowledge comes from premises introduced explicitly or tacitly into the thought experiment. That knowledge is then transformed, usually tacitly, through deductive or inductive argumentation to give the final result.

In Section 3, I will review the case made for this deflationary view of thought experiments. I will elaborate on a recent development that depends on the notion that thought experiments can err but nonetheless can be used reliably. In the guise of the "reliability thesis," I will urge that this is only possible if thought experimentation is governed by a very generalized logic, and evolutionary considerations suggest that these are the logics familiar to us from the literature on deduction and induction. In preparation, in Section 2, I illustrate how thought experiments can err.

In Section 4, I will compare my view with those of my cosymposiasts. James Brown and Tamar Szabó Gendler both urge that thought experiments have more powers epistemically than mere argumentation-they say there is more to the argument. In Sections 4.1-4.3 I will argue that these extra powers, if they exist, would be epistemically irrelevant, since they cannot be used reliably. James McAllister urges that epistemic powers are only accorded to thought experiments when certain narrow, historically contingent presumptions are satisfied. In Section 4.4 I will explain that the mere fact that a science seeks laws is probably sufficient in my view for it to license thought experimentation, and that in turn assures us of the near universal admissibility of thought experiments in science.

2. An Erroneous Thought Experiment. While thought experiments can deliver useful results in science, they are not infallible. Thought experiments can and often do yield false results. This can be seen most clearly in the existence of pairs of thought experiments that give contradictory results. I have described some of these in my (Norton 2004), where I call them thought-experiment/anti-thought-experiment pairs. We can have thought experiments that show the world finite or infinite; that there is an absolute space, or not; that the geometry of a rotating disk is Euclidean, or not; and that an infinite, resting rotor still supplies lift, or not. Here is another example. It is a variant of a well known thought experiment discussed in Brown (1991, 38-40) concerning the Lorentz contraction in the length of moving bodies in special relativity.

Imagine a flat metal plate out of which we carefully cut a long rod that just fits nicely into the slot created, as in Figure 1.

We transport the slotted plate and rod to a region of space remote from gravitation, where they float at rest. We locate the rod above the plate and to one side. We then set the rod into uniform motion towards the slot and in such a way that the rod remains aligned with the slot and parallel to it (see Figure 2). Viewing the process from the plate and slot, 


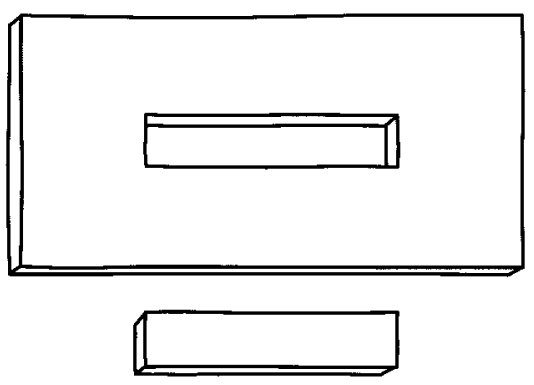

Figure 1. Rod cut from plate leaves slot of same size.

we ask whether the rod will still fit through the slot. According to special relativity, the rod shrinks in the direction of its motion. Since part of that motion lies along the slot, the rod will become shorter than the slot and will pass through easily, as shown in Figure 2.

We now view exactly the same process from the rod. According to that view, the rod remains at rest, but the slot moves uniformly towards it. Aside from the rod and slot switching places, the two cases are exactly the same. At rest the rod and slot are two objects of identical shape and size; all we change is that we move the slot instead of the rod. So this time the slot has shrunk and is shorter than the rod. Therefore the rod cannot pass through the slot.

Something has clearly gone very wrong, for these are two views of the same process. Either the rod passes through the slot or it doesn't. We cannot have both. There is a common and natural response to thought experiments like this one in special relativity. There is only one dependence of length on motion that won't produce problems in a rod-slot thought

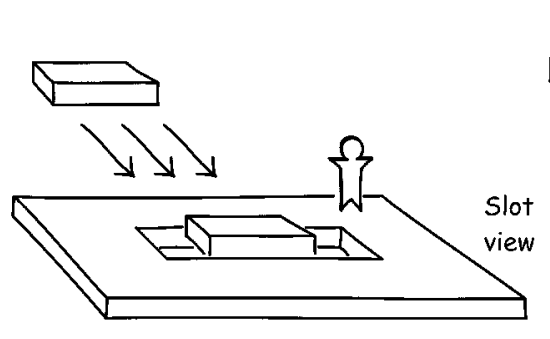

Contracted rod passes through slot.

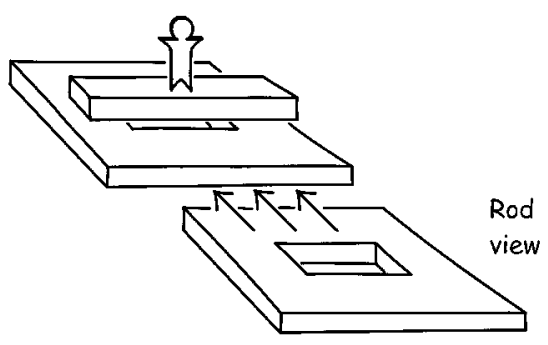

Contracted slot does not pass rod.

Figure 2. Rod and slot in uniform, oblique motion. 
experiment and others like it. It is that the length of a rod does not alter with its motion after all. In that one case, if the resting rod fits the slot exactly when both are at rest, then they will always fit, whether we view a moving rod approaching a resting slot or a moving slot approaching a resting rod. That is our outcome: the relativistic contraction is not real after all. Moving rods and slots don't really shrink.

However one approaches it, this thought experiment manifests error. Indeed Einstein began to fight this attempt at eliminating the Lorentz contraction as early as 1911 (Einstein 1911). How might we characterize the error? We might think of the rod view and slot view as two related thought experiments. Since they disagree on the outcome, at least one must err. They are a thought-experiment/anti-thought-experiment pair since they deliver contradictory outcomes. Or, if one believes that standard special relativity provides a consistent account of moving bodies, the contradictory outcome of the combined thought experiment cannot be right.

\section{Why Arguments?}

3.1. Two Parts of the Thesis Justified. I believe that thought experiments in science are merely picturesque argumentation. I have tried to make my case by dividing the claim into two parts (Norton 1991, 1996, 2004). In brief:

First I urge that thought experiments in science can always be reconstructed as arguments based on explicit or tacit assumptions that yield the same outcome. One basis for this is empiricism. If thought experiments are to teach us about the world, empiricism tells us that they can only do so by drawing on our experience of the world. The result of a thought experiment must be the reformulation of that experience by a process that preserves truth or its probability, that is, by deductive or inductive argumentation. A second, independent basis is that I have found no thought experiment in science that cannot be reconstructed as an argument. Examples include those offered in the literature as resistant to such reconstruction.

Second, I urge that the actual conduct of a thought experiment consists of the execution of an argument. Of course it is very tempting to hold some hybrid view that thought experiments exploit a mysterious mental power whose products can be validated separately by argumentation. That weaker view amounts to a failure to accept the real significance of a remarkable fact: the epistemic reach of a thought experiment coincides exactly with that of an argument. Imagine an oracle that claims mysterious powers but never delivers predictions that could not be learned by simple inferences from ordinary experience. We would not believe that the oracle had any mysterious powers. I propose the same verdict for thought ex- 
periments in science. They have the same epistemic reach as arguments simply because they are arguments.

3.2. The Reliability Thesis. In Norton (2004) I support my view that thought experiments are arguments with:

Reliability thesis. If thought experiments can be used reliably epistemically, then they must be arguments (construed very broadly) that justify their outcomes, or reconstructible as such arguments.

As I will explain below, the thesis draws on a notion of argumentation much broader than the one usually invoked in logic texts.

In brief, the thesis proceeds from the fact that thought experiments can yield erroneous results. Nonetheless we do have confidence in the product of a thought experiment if the thought experiment is properly formulated. But how are we to know that the thought experiment is properly formulated? There must be some sort of mark of truth. The mark is not something external to the thought experiment. It may be comforting to know that Einstein authored the thought experiment. But in principle it should stand alone. We should be able to know that the thought experiment is properly formulated merely by reading its text. So the mark must be something that we can recognize in the thought experiment itself. It cannot merely be that the thought experiment is found in an approved list; that would be an external mark. There must be some systematization - some common feature, some identifiable form, some common structure - in admissible thought experiments whose presence can be perceived by us. That form or structure need not fix all the details of the thought experiment. Indeed it cannot. Newton's bucket thought experiment is clearly unaffected by the choice of a wooden or a leather bucket. So a good thought experiment has two parts: a structure or form that assures its correctness and a portion we can alter without affecting that structure.

That is, a good thought experiment is built from a template into which we are free to insert particular material of our choosing. A familiar example of the use of such templates is in logic: the schemas of a logic are the templates and the sentences or terms inserted into them the variable content. Thus I will say that a generalized logic is what governs any exposition that uses templates with variable content. The range of templates conceivable is enormous. Those employed in thought experiments, however, must be narrower. They must be such that they manifestly support the epistemic aspirations of the thought experiment. The schemas of logic satisfy this requirement in so far as they preserve truth or its probability. Perhaps also we should expect the templates of thought experiments to be sufficiently simple to allow their use to be tractable.

What might the generalized logic of a good thought experiment look 
like if it is not a familiar logic? Nersessian (1993) and Palmieri (2003) have analyzed thought experiments as the manipulation of the mental models of cognitive science. For example, these models would license conclusions about four bodies A, B, C, and D, arranged in a square, by requiring them to conform to the template
A B
C D

As indicated in Norton (2004) I do not think that mental modeling can accommodate all thought experiments. Some, most notably in the physical sciences, depend upon explicit derivations of mathematical results within a physical theory and are unequivocally arguments. I have also yet to see a thought experiment in science (as opposed to other instances of cognition) that cannot be reconstructed as an argument. So I persist in believing that thought experiments are arguments that exploit the familiar deductive and inductive logics.

My confidence in the latter derives from a confidence in the ingenuity of logicians both deductive and inductive, whose profession seeks to extract and codify the schemas used in successful argumentation. Since the activity of thought experimentation has been prominent and important in science for a long time, my view is simply a vote of confidence that the logicians have succeeded in extracting the logics they employ. I do not maintain that thought experiments are arguments because of some belief in the self-discipline of thought experimenters to restrict themselves to a narrow canon of logic. Rather I have confidence that logic evolves to embrace any new, good argument forms that may emerge from the creative efforts of thought experimenters.

4. Epistemologies of Thought Experiments Compared. My cosymposiasts offer different epistemologies of thought experiments. Two are more optimistic than mine over the epistemic powers of thought experiments, and a third more pessimistic. I will use the rod and slot thought experiment as test case to show why I do not share the optimism of the first two. A viable epistemology must give some account of why this thought experiment errs and how thought experiments can still be used reliably if such errors are possible. I will show my argument view can do this but the more optimistic views cannot.

4.1. The Argument View. The error of the rod and slot thought experiment is readily diagnosed and mended by looking at it as an argument. We simply find that it harbors a false assumption. Once that assumption is exposed and corrected, the contradiction with special relativity evaporates. The false assumption is manifested most vividly in Figure 2. We 

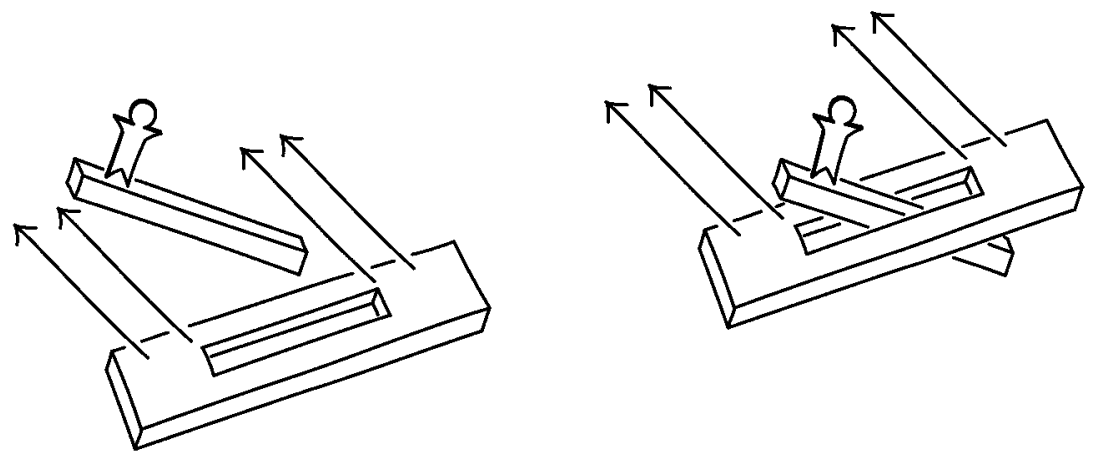

Figure 3. Corrected rod view.

presumed that the rod and slot were parallel when seen in the slot view. The analysis tacitly but erroneously assumed that they remain parallel when we adopt the rod view. In the rod view, they do not remain parallel. The change of view is effected by a Lorentz transformation and this transformation, it turns out, rotates bodies that are oriented obliquely to the direction of motion, such as the rod and the slot. In the corrected rod view, as shown in Figure 3, the rotated rod and slot are no longer parallel and the rotations allow the shorter slot to pass the longer rod. Both views now give the same result: the rod passes.

The argument for the original thought experiment is:

1. A rod moving towards the slot as in Figure 2 (slot view) is contracted and passes through. (Assumption from special relativity)

2. The process viewed from the rod is the same, but with rod and slot switched. (Symmetry assumption)

3. Under the symmetry of 2, if the rod passes in the slot view, it cannot pass in the rod view. (Established in text of thought experiment)

4. The slot does not pass the rod. (From 1, 2, and 3)

5. Contradiction. The rod passes (from 1) and does not pass (from 4).

A second argument reveals what is to be discarded to eliminate the contradiction.

6. There is a modified kinematics that gives consistent results for both views. (Assumption)

7. No dependence of length on motion is the one case which gives consistent results for both slot and rod views. (Assumption)

8. There is no length contraction with motion. (From 6,7)

What we now see is that there is no symmetry between the two views. If 
the rod and slot are parallel in one view, they will not be in the other. And this difference is the essential difference that allows both views to yield the same result. That is, to eliminate the contradiction, we should have discarded the Symmetry Assumption 2, not Lorentz contraction. The argument fails because of a false assumption, 2 .

4.2. Brown's Platonism. Elsewhere (Norton 1993, 1996, 2004) I have described both my admiration for the boldness of James Brown's (1991, 1993a, 1993b, 2004b) Platonic account of certain thought experiments and my misgivings about it. The latter are based in my own skepticism about the reality of a Platonic world of laws that might be accessed by thought experiments or by any other means. Certainly I do not believe that a viable epistemology of thought experiment requires it. The more immediate problem is how thought experiments can be used reliably if they are supposed to be the glimpsing of this Platonic world.

Brown holds that only certain, specially favored thought experiments give us direct access to the Platonic world. They are "Platonic thought experiments" (1991, 43-45). They are distinctive in being simultaneously destructive and constructive; they destroy one view and simultaneously establish another. The rod and slot thought experiment is just such a case. It destroys the kinematics of special relativity, in so far as it shows the Lorentz contraction to be untenable. At the same time, it establishes its replacement, that there is no motion dependent contraction of bodies. Yet it errs. Our Platonic vision was clouded. How are we to know which are the good Platonic thought experiments?

Brown's response is to suggest that ordinary vision can sometime err as well. But we do not dismiss all visual experience as illusion. We can retain some confidence in visual experience, even with only a rudimentary understanding of its mechanism. (1991, 65-66; 1993b) My response is that we do not even have the most rudimentary account of the nature of Platonic perception (Norton 2004). ${ }^{1}$ We might try to correct its products by noting that the outcome of the rod and slot thought experiment contradicts special relativity. But how can we be so sure that this isn't the thought experiment that seals the fate of special relativity after all? My answer is simple. We recognize that thought experiments are merely arguments and this particular one harbors a false assumption. Brown may want to point out the same false assumption to explain the failure of Platonic vision. But in doing this he is simply replicating the argument-

1. Indeed as far as I can tell nothing precludes argumentation itself being the vehicle for accessing the Platonic world. If that were the vehicle, there would be no way in principle to distinguish Brown's account from mine. I would then claim that mine is preferable on grounds of simplicity. 
based analysis just given. Nothing in the diagnosis depends on thought experiments being anything more than arguments.

Brown (2004a) has offered an ingenious thought experiment that purports to disprove the continuum hypothesis. Contrary to Brown's suggestion, it can be reconstructed as an argument whose crucial early stages are:

1. The probability of a dart hitting a region of the dart board is proportional to its area. (Assumption)

2. Therefore, the probability of hitting the infinitely thin, zero-area wires on the dart board is zero. (From 1)

3. A measure-zero set of reals is analogous to infinitely thin wires and random selection of reals is analogous to dart throws. (Assumption)

4. Therefore, the probability of randomly selecting a real from a measure-zero set of reals is zero. (From 2, 3, by analogy)

5. If $f$ is any function from the reals to countable sets of reals, $x$ some real, and $y$ a randomly chosen real, then with probability $1, y$ is not in $f(x)$. (From 4)

This last conclusion delivers the first part of the Freiling Symmetry Axiom (FSA), which is equivalent to the negation of the continuum hypothesis.

While it is immaterial to the issues that separate us, I believe that it is impossible to recover the remainder of FSA in the thought experiment, whether by ordinary argumentation or Platonic vision. Let us say that the "dart principle" assures us that there is a probability zero of choosing a real from a measure-zero set. This assures us that, for some fixed $f$, we can always pick pairs of reals $x, y$ so that $y$ is not in $f(x)$, where $f$ is any function that maps reals to countable sets of reals. But FSA requires more. It requires each such function $f$ to satisfy the dual property:

$y$ is not in $f(x)$ and $x$ is not in $f(y)$.

Conjoining the two seems harmless enough. As Brown stresses, there is a symmetry in the selection of $x$ and $y$, so why not? The supposed harmlessness depends on the function $f$ respecting that symmetry, in the sense that the direct property ( $y$ is not in $f(x)$ ) does not interfere with the inverse property ( $x$ is not in $f(y)$ ). As it turns out, there are pathological functions which do not respect this symmetry. For them, the satisfaction of the direct property guarantees the failure of the inverse property. Brown's discussion contains an example: $f(x)=\{y: y \leq x\}$ where $<$ is a well-ordering of the reals.

How is this possible? It arises because once the dart principle is used to justify the direct property, it can no longer justify the inverse property. To use the dart principle for the direct property, we presume a fixed $x$ and note that any $y$ outside the measure-zero set $f(x)$ suffices. For the 
resulting pair to satisfy the inverse property, the $y$ we choose must have the additional property of not being in the set $X=\{z: x$ is in $f(z)\}$. This set $X$ need not have measure zero and may have measure one. So the dart principle cannot assure us that such a $y$ can be found. Therefore it cannot assure us of FSA.

4.3. Gendler's Constructivism. Tamar Szabó Gendler (1998) has elaborated a view of thought experiments that derives from Kuhn ([1964] 1977). The principal notion is that a function of a thought experiments is to reveal lacunae in conceptual systems and to indicate how they are to be altered. I am very largely in agreement with her analysis. That function of thought experiments is both interesting and important. Where we differ, however, is in her insistence that their serving of this function shows thought experiments cannot be arguments. I am far more optimistic about the power of argumentation to reconfigure conceptual schemes. The argument of Russell's paradox led to the overthrow of the naïve conception of sets. The relative consistency proofs of the nineteenth century placed Euclidean and non-Euclidean geometry on equal footings as far as consistency is concerned. That eradicated millennia of belief that there is something logically defective about a geometry that violates Euclid's postulates. Or Gödel's proof of the incompleteness of arithmetic showed us that a concept of truth as derivability is inadequate. This is not a bad showing for mere argumentation.

Gendler seems to allow that mere argumentation can reveal a contradiction. But she suggests that it cannot tell us what to adjust in our conceptual scheme in order to eliminate the contradiction. Here again I agree. The decision of what is refuted by a reductio ad absurdum seems to depend largely on the way the argument is set up and the predilections and larger interests of the scientist. However I have yet to see that thought experiments do any better in this task. The context of the thought experiment may powerfully suggest the target for the reductio and it may just be completely wrong. Such is precisely the case in the rod and slot thought experiment. We generate a contradiction, so we know we are mistaken somewhere. The setting of the thought experiment almost irresistibly draws us to the wrong target, the Lorentz contraction. The real culprit, the symmetry assumption, escapes because it is so natural, even though it rests on well-disguised assumptions about absolute simultaneity that are inadmissible in special relativity.

If thought experiments have some extra power to reveal the right culprit when a contradiction emerges, what is the epistemic basis of this power? Gendler has mentioned Mach's idea of tacit knowledge assimilated from experience and talks of a "constructive participation" by the reader (414415). How are we to know when the power of constructive participation 
is exercised well? How are we to distinguish it from those cases like the rod and slot in which the thought experiment proves to be massively misleading in this aspect? In short, if thought experiments have such extra powers, how can they be exercised reliably?

4.4. McAllister and the Evidential Inertness of Thought Experiments in Science. While Brown and Szabó Gendler are considerably more optimistic than I on the epistemic power of thought experiments, my cosymposiast James McAllister (1996) has developed an unwarranted pessimism. He observes correctly that thought experiments can only have persuasive power if they play by the right epistemic rules. The ritualistic reading of entrails, for example, does not play by these rules and thus has no epistemic power in science. However he thinks the epistemic license for thought experiments is narrow and time dependent. His sustained example is a "Galilean doctrine of phenomena" which licenses thought experimentation on accident-free phenomena whose behavior is idealized as sufficiently regular to be subject to laws. The familiar example is bodies falling freely without the accidental complications of air resistance. Aristotelians concern themselves with real occurrences with all their accidents in place and thus, on McAllister's account, eschew Galileo's thought experiments on falling bodies.

All I believe McAllister can show is that Aristotelians eschew particular thought experiments that may depend upon a notion they find illicit. He does not show they eschew thought experimentation in general. And he cannot. Thought experiments appear throughout Aristotle's corpus. ${ }^{2}$ Some are of cosmic moment and do even deal with the fall of bodies. Aristotle (On the Heavens, 297a13-30) imagines the earth in formation and recounts how the motion of bodies to the center would yield a spherically shaped earth. Indeed, in a thought experiment of breathtaking ambition, Aristotle continues (297a33-297b13) to imagine that a weight many times that of the earth is added to one half of the earth's sphere and then traces out the resulting motions.

McAllister's concentration on the Galilean doctrine of phenomena as the grounding of thought experiment is entirely too narrow. ${ }^{3}$ Worse his

2. I am grateful to James Lennox for drawing my attention to two thought experiments in Aristotle's biology in Progression Animals, 708b4-10 and 709a1-8 pertaining to the locomotion of animals.

3. McAllister (1996, section 6) has also urged that there cannot be thought experiments that shed light on the nature of discontinuous quantum state transitions - "jumps"because their indeterministic character does not admit Galilean phenomena. It is not clear to me that their indeterministic character does preclude Galilean phenomena; and, if it does, it is not clear to me that this is sufficient to rule out thought experimentation; and it is not clear to me that there are not such thought experiments. Bohm 
insistence $(1996,242)$ that the evidential power of thought experiments is conferred "by the persuasive effort of scientists" confuses matters by conflating sociological facts about scientists with what is licit in their theories or epistemologies. McAllister alludes to "some alternative rationale" (248). I believe that they are available copiously. Thought experiments are just picturesque argumentation of a hypothetical or counterfactual nature. Essentially all that is needed is that the science admit hypothetical or counterfactual reasoning for it to admit thought experimentation. It is by no means assured that a field of inquiry will admit such reasoning. In many historical circles, counterfactual histories are simply dismissed as illicit; historical scholarship does not support counterfactuals, we are told. However it is hard to imagine a science that does not admit such reasoning. All it must do is seek laws or even just projectible regularities; these support hypotheticals or counterfactuals; and these in turn support thought experiments. That doesn't mean the scientist must actually conduct thought experiments. They just might not be useful. But if the science supports counterfactuals, they are admissible. For example, the classification systems of botany sustain counterfactuals and thus admit thought experiments, although there seem to be few. Linnaeus, founder of modern botany, remarks $(1786,58)$ that the modern diversity of species of vegetables results from hybridization not from changes of soil, "else the plants would return to their original form, if removed again to their original situation." What of a very impoverished view that sees science merely as cataloguing occurrent fact and never going beyond it? Might that view preclude thought experimentation? Curiously Mach's own positivism came closest to this view, but even he did not develop it to the point that thought experimentation was precluded. His careful reflections on the topic established the modern literature in thought experiments.

\section{REFERENCES}

Bohm, D. ([1951] 1989), Quantum Theory. Englewood Cliffs, NJ: Prentice-Hall. Reprinted Mineola, NY: Dover.

Brown, James Robert (1991), The Laboratory of the Mind: Thought Experiments in the Natural Sciences. London: Routledge. 279.

(1993a), "Why Empiricism Won't Work", in Hull, Forbes, and Okruhlik 1993, 271- $(199$

40.

(2004a), "Peeking into Plato's Heaven”, Philosophy of Science 71 (Proceedings): $1126-1138$.

(2004b), "Why Thought Experiments Transcend Experience", in Christopher Hitchcock (ed.), Contemporary Debates in Philosophy of Science. Oxford: Blackwell, 23-43.

$(1951,107)$ imagines a pulse of light generated by a shutter and then absorbed by an atom in a quantum jump. The thought experiment shows the energy $\Delta E$ and time $\Delta t$ of the jump are governed by the uncertainty relation $\Delta E \cdot \Delta t \geq h / 2 \pi$. 
Einstein, Albert (1911), “Zum ehrenfestschen Paradoxon”, Physikalische Zeitschrift 12: 509 510 .

Gendler, Tamar Szabó (1998), "Galileo and the Indispensibility of Scientific Thought Experiment", The British Journal for the Philosophy of Science 49(3): 397-424

Horowitz, Tamara, and Gerald J. Massey (eds.) (1991), Thought Experiments in Science and Philosophy. Savage, MD: Rowman and Littlefield.

Hull, David, Micky Forbes, and Kathleen Okruhlik (eds.) (1993), PSA 1992, vol. 2. East Lansing, MI: Philosophy of Science Association.

Kuhn, T. S. ([1964] 1977), "A Function for Thought Experiments," in L'aventure de la science: Mélanges Alexandre Koyré, vol. 2, Paris: Hermann, 307-334. Reprinted in The Essential Tension: Selected Studies in Scientific Tradition and Change. Chicago: University of Chicago Press, 240-265.

McAllister, James (1996), "The Evidential Significance of Thought Experiment in Science", Studies in History and Philosophy of Science 27: 233-250.

Nersessian, Nancy (1993), "In the Theoretician's Laboratory: Thought Experimenting as Mental Modeling", in Hull, Forbes, and Okruhlik 1993, 291-301.

Norton, John D. (1991), "Thought Experiments in Einstein's Work", in Horowitz and Massey 1991, 129-148.

(1993), "Seeing the Laws of Nature" (review of Brown 1991), Metascience 3 (new series): $33-38$

(1996), “Are Thought Experiments Just What You Thought?", Canadian Journal of Philosophy 26(3): 333-366.

(2004), "Why Thought Experiments Do Not Transcend Empiricism", in Christopher Hitchcock (ed.), Contemporary Debates in the Philosophy of Science. Oxford: Blackwell, 44-66.

Palmieri, Paolo (2003), "Mental Models in Galileo's Early Mathematization of Nature", Studies in History and Philosophy of Science Part A 34(2): 229-264.

Linnaeus, C. (1786), A Dissertation on the Sexes of Plants. Translated by James Edward Smith. London. 